\title{
Acoso escolar y apego familiar: la perspectiva del alumnado agresor
}

\section{Bullying and family attachment: Bully students' perspectives*}

\author{
IRATXE URRESTI-PADRÓN ${ }^{1}$ \\ iratxeurrestipadron@hotmail.es \\ LUIS FELICIANO-GARCÍA \\ Ifelici@ull.edu.es \\ Lidia E. Santana-Vega \\ Isantana@ull.es \\ Universidad de La Laguna, España
}

\section{Resumen:}

El acoso escolar es un fenómeno que tiene lugar en todo el mundo y constituye una gran preocupación para los sistemas educativos de los países desarrollados y en vías de desarrollo. El presente estudio analiza: a) las diferencias en el perfil de agresión del alumnado de Educación Secundaria Obligatoria en base a variables personales, académicas y familiares; y

b) las diferencias en la valoración de la gravedad de conductas agresivas según el perfil de agresión del alumnado. En la investigación participaron 215 alumnos/as de Educación Secundaria Obligatoria de dos centros educativos de Tenerife. Para la recogida de datos se diseñó un cuestionario en el que se abordaron: 1) los niveles de agresión del alumnado; 2) la calidad de las relaciones del alumnado con sus padres y madres; y 3) la gravedad atribuida por el alumnado a situaciones de agresión. Los resultados reflejaron que los estudian-

\section{Abstract:}

Bullying is a phenomenon that happens worldwide and a major concern for the educational systems in developed and developing countries. The present study analyzes: a) the differences in the aggression profile of Compulsory Secondary Education students based on personal, academic and family variables; and b) the differences in the assessment of the seriousness of students' aggressive behaviors according to the students' aggression profile. The research involved 215 students of Compulsory Secondary Education from two schools in Tenerife. For the collection of data, a questionnaire was designed that addressed: 1) students' aggression levels; 2) the quality of students' relationships with their parents; and 3) the seriousness which students attributed to aggression situations. The results showed that the students with the highest aggression scores were: a) males; b) repeaters; c) those

* El objetivo es analizar la visión/la perspectiva del alumnado agresor, considero que se entiende mejor la idea en singular.

1 Dirección para correspondencia (correspondence address):

Iratxe Urresti-Padrón. Departamento de Didáctica e Investigación Educativa. Facultad de Educación. C/ Pedro Zerolo, s/n. Edificio Central. Planta 2. Apartado 456. 38200, San Cristóbal de La Laguna. Santa Cruz de Tenerife (España). 
tes con mayores puntuaciones en agresión son: a) varones; b) repetidores/as; c) aqueIlos/as cuyos padres poseen un menor nivel educativo; d) aquellos/as que tienen mayor nivel de alienación con padres y madres; y e) aquellos/as que tienen menor nivel de confianza con su padre. Los estudiantes que muestran mayor nivel de agresión tienden a valorar las conductas agresivas como menos graves que quienes muestran menor nivel de agresión. Se concluye que determinadas características socio-familiares en los jóvenes, como la confianza con los padres y el sentimiento de integración con ambos progenitores, actúan como factores protectores ante el acoso escolar.

\section{Palabras clave:}

Acoso; agresión; conducta de apego; relaciones familiares. whose parents have a lower educational level; d) those who have higher levels of alienation with fathers and mothers; and e) those who have a lower degree of closeness with their father. Students who have a higher level of aggression tend to value aggressive behaviors as less serious than those who have a lower level of aggression. It is concluded that certain socio-family characteristics in young people, such as closeness with parents and the feeling of integration with both parents, act as protective factors against bullying.

\section{Keywords:}

Bullying; aggression; attachment behaviour; family relationships.

\section{Résumé:}

Les brimades sont un phénomène mondial et constituent une préoccupation majeure pour les systèmes éducatifs des pays développés et en développement. Cette étude analyse : a) les différences dans le profil d'agression des élèves de l'enseignement secondaire obligatoire en fonction de variables personnelles, académiques et familiales; b) les différences dans l'évaluation de la gravité des comportements agressifs en fonction du profil d'agressivité des élèves profil agressif des étudiants. La recherche a porté sur 215 élèves de l'enseignement secondaire obligatoire de deux écoles. Enseignement secondaire obligatoire dans deux écoles de Tenerife. Afin de collecter les données, un questionnaire a été élaboré. un questionnaire a été conçu pour collecter des données sur : 1) les niveaux d'agressivité des étudiants; 2) la qualité des relations des élèves avec leurs parents; et 3) la gravité attribuée par les élèves aux situations agressives. Les résultats ont montré que les élèves ayant. Les résultats ont montré que les élèves ayant des scores d'agressivité plus élevés sont : a) les garçons; b) les redoublants; c) ceux dont les parents ont un niveau d'éducation plus faible; d) ceux qui ont un niveau plus élevé d'aliénation avec les parents ; et e) ceux qui ont un niveau plus élevé d'aliénation avec les parents. avec les pères et les mères. Le site les élèves qui manifestent un niveau d'agressivité élevé ont tendance à considérer les comportements agressifs comme moins graves que ceux qui manifestent un niveau d'agressivité plus faible. II est conclu que certaines caractéristiques socio-familiales des jeunes, telles que la confiance avec les parents et le sentiment d'intégration avec les deux parents et le sentiment d'intégration avec les deux parents, agissent comme des facteurs de protection contre les brimades.

Mots clés:

Intimidation; agression; comportement d'attachement; relations de famille.

Fecha de recepción: 09-01-2020

Fecha de aceptación: 22-02-2020 


\section{Introducción}

Los comportamientos de acoso no son algo inherente a la sociedad actual, son tan antiguos como las propias relaciones humanas (Nuñez, Herrero y Aires, 2006). Sin embargo, en los últimos años, el acoso escolar se ha convertido en una importante preocupación social debido a las graves consecuencias negativas que conlleva para los/as involucrados/as (Murphy, Laible \& Augustine, 2017). Este fenómeno se ha visto agravado por la aparición de nuevas formas de "hostigar al otro" relacionadas con las nuevas tecnologías y las redes sociales (Pulido y Tarancón, 2018).

El reconocimiento de la existencia de conflictos dentro de las escuelas ha provocado que se incrementen los estudios sobre las formas en que estos se manifiestan (Valdés, Martínez y Torres, 2012). Si bien en las últimas décadas se han llevado a cabo numerosas investigaciones a nivel nacional e internacional sobre este fenómeno escolar, son escasas las realizadas en la comunidad autónoma de Canarias. Por ello, este trabajo pretende abordar el estudio del acoso escolar desde la perspectiva del alumnado que cursa sus estudios de Educación Secundaria Obligatoria en Tenerife. Debido al papel fundamental de las familias en la limitación o potenciación de los conflictos escolares, es necesario analizar la relación entre las dinámicas familiares y la implicación del alumnado en situaciones de acoso dentro de la escuela. Los objetivos del estudio son:

a) Analizar las diferencias en el perfil de agresión del alumnado en base a variables personales y académicas.

b) Analizar las diferencias en el perfil de agresión del alumnado en base al estilo de apego con sus padres y madres.

c) Analizar las diferencias en la valoración de la gravedad de conductas agresivas según el perfil de agresión del alumnado.

\section{Marco empírico}

Para que una conducta pueda ser entendida como acoso debe conllevar voluntad, desequilibrio y persistencia. El primero de estos criterios hace referencia al carácter intencional del/a agresor/a por hacer daño -no necesariamente físico- a la víctima. El segundo encuadra el acoso en el marco de una relación de desequilibrio de poder y un esquema de 
dominio-sumisión entre agresor/a y víctima. Esta distorsión de la reciprocidad esperable entre iguales hace que la víctima no tenga recursos psicológicos suficientes para parar la agresión o, al menos, enfrentarse a ella (Falla y Ortega-Ruíz, 2019). El tercer criterio hace referencia al carácter sistemático, reiterativo y repetitivo de la conducta de acoso. Por lo general, no se denomina acoso a un episodio aislado de agresión o violencia.

Centrándonos en el acoso escolar, Olweus (1997), autor pionero en investigaciones sobre este fenómeno (1978, 1999), lo definió como un comportamiento desagradable, repetitivo e intencional que ocurre entre los miembros de la comunidad educativa y está dirigido contra una o más personas que tienen dificultad para defenderse. El tipo de acoso escolar más grave es el que sucede entre iguales. Este puede llevarse a cabo de manera directa, con ataques abiertos a la víctima, o de manera indirecta, aislando socialmente a la víctima y excluyéndola deliberadamente del grupo (Nuñez et al., 2006).

En España, las cifras de acoso escolar han ido aumentando los últimos años, alcanzando más de 1000 casos denunciados en 2017. Andalucía es la comunidad autónoma con mayor número de víctimas de acoso escolar anuales (255), seguida de Madrid (170), Comunidad Valenciana (129) y Canarias (100) (Ministerio de Educación, Cultura y Deporte, 2019).

En las situaciones de acoso escolar, el alumnado puede presentar tres tipos de roles: agresor/a, víctima y espectador/a. Existen otros agentes implicados como la familia, el personal docente y el personal no docente de los centros educativos.

El rol de agresor/a, especialmente, está ligado a estudiantes con baja competencia emocional y escasas habilidades para la resolución de conflictos, lo que tiene como consecuencia una baja popularidad entre los/ as compañeros/as (Olweus, 1998). Los/as agresores/as tienen el deseo de tener el control y la necesidad constante de reconocimiento social; por lo que, paradójicamente, presentan una alta dependencia con respecto a sus pares.

Este tipo de alumno/a suele tener problemas en el ámbito escolar ya que tiende a mostrar comportamientos desafiantes, no respetar las normas sociales y mantener malas relaciones con las figuras de autoridad. Además, muestra un alto rechazo a la escuela, bajo rendimiento académico y, consecuentemente, alto fracaso escolar (Díaz-Aguado, 2005; 
Rodrigo, Máiquez, Batista-Foguet, García, Rodríguez, Martín y Martínez, 2006).

El alumnado agresor no es capaz de "ponerse en el lugar" de los/as demás, muestra poca sensibilidad ante el dolor ajeno y escasa empatía, por lo que no siente culpabilidad cuando agrede a sus víctimas (Nolasco, 2012; Olweus, 1998). Estos estudiantes no son autocríticos; no suelen percibirse a sí mismos/as como agresores/as, se justifican y creen que solo se defienden ante las agresiones o provocaciones de sus víctimas (Díaz-Aguado, 2005; Serrano e Iborra, 2005). Otras características distintivas de los/as agresores/as son impulsividad, baja tolerancia a la frustración y al fracaso y mayor tolerancia hacia la violencia (Díaz-Aguado, 2005; Ulloa, 2015). Así, generan intimidación, exclusión y, en ocasiones, serios problemas psicológicos y sociales a las víctimas, provocando dificultades en la convivencia escolar (Castillo-Pulido, 2011).

El acoso escolar es más propenso en chicos que en chicas (Serrano e Iborra, 2005; Oñate y Piñuel, 2007; González-Cabrera, Tourón, Machimbarrena, León-Mejía y Gutiérrez- Ortega, 2019), especialmente en los niveles correspondientes a la Educación Secundaria (Olweus, 1998). Entre los chicos son más habituales las agresiones físicas y verbales; mientras que las chicas suelen emplear formas más sutiles e indirectas de acoso, tales como exclusión, intimidación psicológica, invención de rumores y manipulación. Las relaciones entre los varones tienden a ser más agresivas, teniendo estas diferencias raíces tanto biológicas como socio-ambientales (Olweus, 1997).

Los/as alumnos/as que han repetido algún curso se ven más implicados en el acoso escolar como acosadores/as, en conductas como insultar, amenazar, pegar, intimidar, poner motes, reírse de otros/as, ignorar y no dejar participar a otros/as compañeros/as en las actividades grupales. Destaca el gran porcentaje de exclusión social y rechazo que sufre el alumnado escolarizado en grupos de edad inferior a la suya, recayendo en él el estigma negativo de haber repetido curso; lo que le impide la integración total con el grupo-clase (Enríquez, 2015; Medel, 2017). Se pone de manifiesto la asociación entre factores individuales y sociales a la hora de comprender y abordar el acoso escolar (Sánchez, 2009).

En la adolescencia, los jóvenes se enfrentan a intensas demandas (Araujo, Gomes, Da Luz y Medeiros, 2012). Entre ellas, se encuentra la necesidad de afrontar la transición entre la educación primaria -más personalizada y con un grupo social sólido- y la educación secundaria -más 
impersonal y caracterizada por una menor supervisión del alumno/a-. Es en este periodo donde las relaciones sociales entre iguales cobran especial importancia y se comienza a utilizar la agresividad para establecer una posición social respecto al nuevo grupo generado a raíz de esa transición escolar (Pellegrini \& Long, 2002).

Durante la etapa vital de la adolescencia, acontece una evolución, no solo en las relaciones del alumnado con los iguales, también con la familia (Fernández, Goñi, Camino y Ramos, 2019; Santana, 2013; Santana, Feliciano y Jiménez, 2012). Las interacciones que tienen lugar dentro del sistema familiar juegan un papel importante en la socialización de los/as niños/as y sus resultados psicosociales (Demby, Riggs \& Kaminski, 2017), así como en el impacto de las situaciones de acoso escolar.

La comunicación familiar abierta y empática es un factor clave; aqueIlos/as alumnos/as que pueden mantener conversaciones con sus padres/ madres sobre su vida generan soluciones más efectivas ante las situaciones sociales. La capacidad de comunicarse con los progenitores fomenta una mayor comprensión de los demás, produciendo una reducción en los comportamientos de intimidación (You, Lee, Lee \& Kim, 2015). La falta de conversaciones y la imposibilidad de discutir los problemas con los padres y madres provoca mayores niveles de acoso (Shetgiri, Lin, Avila \& Flores, 2012).

El estilo de apego entre progenitores e hijos/as se establece de forma temprana durante las primeras relaciones, permaneciendo relativamente estable durante la adolescencia y el resto de la vida adulta. Formas de apego poco adaptativas, como el apego evitativo y el apego inseguro, correlacionan con conductas de acoso (Balan, Dobrean \& Balazsi, 2018; Magaz, Chorot, Sandin, Santed y Valiente, 2011; Murphy et al., 2017). El estilo de apego seguro, la capacidad de respuesta afectiva de los progenitores y altos niveles de conexión y apoyo familiar son fundamentales para afrontar los desafíos que presenta la adolescencia, reduciendo las probabilidades de que los/as jóvenes elijan comportamientos de acoso (Bowes, Arseneault, Maughan, Taylor, Caspi \& Moffitt, 2009; Cooper, Shaver \& Collins, 1998; Mann, Kristjansson, Sigfusdottir y Smith, 2015).

Existe una relación entre las prácticas parentales coherentes con el estilo educativo democrático y una mayor probabilidad de desarroIlar un apego seguro entre padres/madres e hijos/as (Cantón y Cortés, 2000; Gómez-Ortiz, Del Rey, Romera, y Ortega-Ruiz, 2015). Es razonable que el estilo educativo parental $y$, particularmente, el control 
conductual también se relacionen con la implicación en el acoso escolar, especialmente en el rol de agresor (Gómez- Ortiz et al., 2014). El estilo educativo parental que más protege de la participación en situaciones de acoso es el estilo democrático, caracterizado por una elevada percepción de afecto parental por parte de los/as jóvenes y moderado-sostenido control de la conducta. Los/as hijos/as de padres y madres con estilos educativos punitivos e indiferentes muestran una elevada puntuación en agresión (Hong, Kim \& Piquero, 2017). Los/as acosadores/as tienden a tener padres/madres que no brindan una supervisión adecuada o que no participan activamente en la vida de sus hijos/as (Espelage, Bosworth \& Simon, 2000; Georgiou \& Fanti, 2010; Low \& Espelage, 2013). El monitoreo parental constante ha sido reconocido durante mucho tiempo como un factor protector para el desarrollo de los/as adolescentes; aquellos/as que declaran un elevado nivel de exigencia paterna/materna y un mayor uso de castigos físicos y psicológicos tienen actitudes más favorables hacia la violencia entre iguales (De la Villa y Ovejero, 2013).

Una buena relación familiar, caracterizada por el apego entre sus miembros, permite a los/as padres/madres ofrecer a sus hijos/as modelos funcionales de relaciones sociales que pueden utilizar en otros contextos de desarrollo, como el escolar. Las dinámicas familiares deben ser objeto de acción en los programas de prevención del acoso escolar. Las intervenciones dirigidas a las familias deben centrarse en el desarrollo de normas y valores que contribuyan al respeto, la confianza, el apego y la consideración de las necesidades de cada uno/a de los/as integrantes del núcleo familiar (Espelage \& Swearer, 2009).

\section{Método}

\section{Participantes}

En el estudio participaron 215 estudiantes que cursaban Educación Secundaria Obligatoria en dos centros educativos ubicados en Santa Cruz de Tenerife (véase Tabla 1). 
Tabla 1

Características de la muestra

\begin{tabular}{|c|c|c|c|c|c|c|c|c|c|c|}
\hline \multicolumn{3}{|c|}{ Titularidad del centro } & \multicolumn{2}{|c|}{ Sexo } & \multicolumn{4}{|c|}{ Curso } & \multicolumn{2}{|c|}{ Factor Repetición } \\
\hline \multicolumn{2}{|r|}{ Público } & $\begin{array}{c}\text { Concertado- } \\
\text { Privado }\end{array}$ & Mujer & Hombre & $\begin{array}{c}1 .^{\circ} \\
\text { ESO }\end{array}$ & $\begin{array}{c}2 .^{\circ} \\
\text { ESO }\end{array}$ & $\begin{array}{r}3 .^{\circ} \\
\mathrm{ESO}\end{array}$ & $\begin{array}{c}4 .^{\circ} \\
\mathrm{ESO}\end{array}$ & Repetidor & $\begin{array}{c}\text { No } \\
\text { repetidor }\end{array}$ \\
\hline $\mathrm{N}$ & 98 & 117 & 106 & 108 & 53 & 56 & 54 & 52 & 22 & 193 \\
\hline$\%$ & 45.6 & 54.4 & 49.5 & 50.5 & 24.7 & 26 & 25.1 & 24.2 & 10.2 & 89.8 \\
\hline \multicolumn{5}{|c|}{ Nivel Educativo Padre } & \multicolumn{6}{|c|}{ Nivel Educativo Madre } \\
\hline & $\begin{array}{l}\text { ucación } \\
\text { maria }\end{array}$ & $\begin{array}{l}\text { Educación B } \\
\text { Secundaria }\end{array}$ & $\begin{array}{c}\text { Bachiller } \\
\text { / FP } \\
\end{array}$ & $\begin{array}{c}\text { Univers } \\
\text { dad }\end{array}$ & $\begin{array}{r}\text { i- Edu } \\
\text { Pri } \\
\end{array}$ & $\begin{array}{l}\text { ucació } \\
\text { imaria } \\
\end{array}$ & $\begin{array}{ll}\text { Edu } \\
\text { Sec }\end{array}$ & $\begin{array}{l}\text { lucación } \\
\text { cundaria }\end{array}$ & 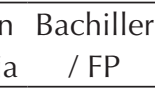 & $\begin{array}{c}\text { Universi- } \\
\text { dad }\end{array}$ \\
\hline $\mathrm{N}$ & 7 & 24 & 44 & 118 & & 4 & & 18 & 54 & 129 \\
\hline$\%$ & 3.6 & 12.4 & 22.8 & 61.1 & & 2 & & 8.8 & 26.3 & 62.9 \\
\hline
\end{tabular}

\section{Instrumentos}

Para obtener la información relativa a los objetivos de la investigación se aplicó un cuestionario. El cuestionario utilizado, además de recabar datos personales y académicos del alumnado, indaga sobre las siguientes dimensiones de información: a) los niveles de agresión del alumnado; b) la calidad de las relaciones familiares del alumnado; y c) la gravedad atribuida por el alumnado a diversas conductas de agresión. El cuestionario incluye escalas diseñadas por otros/as autores/as, seleccionadas por su adecuación para examinar las variables objeto de estudio y por sus características psicométricas:

1) La Escala de Agresión, diseñada por Orpinas y Frankowski (2001) y adaptada al castellano por López y Orpinas (2012), examina el número de conductas de agresión física y verbal llevadas a cabo por el alumnado durante la semana anterior a la aplicación del cuestionario. La escala contiene 11 ítems con siete categorías de respuesta que van desde 0 veces hasta 6 o más veces. Esta escala permite analizar dos factores: agresión física y verbal (conductas que pueden conllevar lesiones físicas y/o psicológicas para las víctimas) y enfado (la emoción más asociada con la agresión). En su versión española, el Alfa de Cronbach indicó un nivel adecuado de consistencia interna $(a=.86)$ y el análisis factorial de los 11 ítems arrojó dos factores que en su conjunto explicaron el $42 \%$ de la varianza total.

2) El Inventario de Apego con Padres y Pares (IPPA), diseñado por 
Armsden y Greenberg (1987), examina la calidad del estilo de apego con padres, madres y pares percibida por el alumnado. En el presente estudio, se utilizaron los 25 ítems relativos al padre y los 25 relativos a la madre. Cada ítem presenta cinco alternativas de respuesta, siendo 1 nunca y 5 siempre. Este inventario permite analizar tres factores: confianza (comprensión, respeto mutuo y confianza mutua), comunicación (calidad de la comunicación verbal con los progenitores) y alienación (sentimientos de aislamiento respecto a los progenitores). En su versión española, el Alfa de Cronbach de las escalas confianza y comunicación fue superior a .83; mientras que el a de la escala alienación osciló entre .54 y .68. El análisis factorial arrojó 3 factores para ambas escalas que explicaron el $46.98 \%$ de la varianza total en la escala de la madre y el $56.36 \%$ de la varianza total en la escala del padre.

Además de estas escalas, el cuestionario incluye una pregunta de elaboración propia en la que se indaga sobre la gravedad que para el alumnado tienen 31 conductas de agresión entre escolares. La gravedad de cada conducta se valora en una escala de 5 puntos, siendo 1 nada grave y 5 muy grave. El estadístico Alfa de Cronbach indicó un nivel muy adecuado de consistencia interna $(\alpha=.90)$. La prueba KMO obtuvo un valor de .93 y la prueba de Esfericidad de Bartlet resultó significativa $(p<0.000)$. El análisis factorial de los 31 ítems mostró cinco factores (discriminación sexual, conductas de autoridad, conductas de agresión leves, burlas y humillación), que en su conjunto explicaron el $60.88 \%$ de la varianza total.

\section{Análisis de datos}

Tras la aplicación de los cuestionarios, se realizó el vaciado de datos y su análisis con el programa estadístico SPSS 21. El análisis de datos comprendió: estadísticos descriptivos para cada una de las variables estudiadas, coeficiente de fiabilidad, análisis factorial, contraste de medias para grupos independientes, ANOVA y d de Cohen para calcular el tamaño del efecto. 


\section{Resultados}

La media de puntuaciones en la Escala de Agresión fue de 11.7 (DT = 11.66), lo que indica que durante la semana previa a la encuesta, los estudiantes participaron en un promedio de casi 12 acciones de agresión. El presente estudio encontró que: a) los varones participaron en más acciones de agresión que las mujeres, con un tamaño pequeño del efecto; b) los estudiantes que han repetido algún curso de Educación Secundaria Obligatoria indicaron haber participado en más acciones de agresión que aquellos que no han repetido ningún curso, con un tamaño medio del efecto; y c) los estudiantes cuyos padres tienen estudios de secundaria informaron haber participado en más acciones de agresión que aquellos cuyos padres tienen estudios universitarios, con un tamaño medio del efecto (véase Tabla 2). No se observan diferencias significativas en el nivel de agresión de los estudiantes en función del curso en el que se encuentran, ni del nivel educativo de la madre.

Tabla 2

Puntuaciones en la Escala Agresión en función de las variables académicopersonales

\begin{tabular}{ccccccc}
\hline & Mujer Hombre Repetidor & $\begin{array}{c}\text { No } \\
\text { repetidor }\end{array}$ & $\begin{array}{c}\text { Padre } \\
\text { Estudios } \\
\text { Secundaria }\end{array}$ & $\begin{array}{c}\text { Padre } \\
\text { Estudios } \\
\text { Universitarios }\end{array}$ \\
\hline Media & 9.25 & 14.04 & 18.96 & 11.00 & 16.36 & 10.21 \\
DT & 9.94 & 13.87 & 15.16 & 11.76 & 13 & 11.5 \\
F & $\mathrm{F}(212)=10.42$ & $\mathrm{~F}(208)=2.8$ & $\mathrm{~F}(2)=3.2$ \\
p & $<.05$ & $<.05$ & $<.05$ \\
d de Cohen & .40 & & .58 & & \multicolumn{2}{c}{.5} \\
\hline
\end{tabular}

En el Inventario de Apego con Padres y Madres, los resultados relativos al padre manifiestan que los/as participantes con mayores niveles de agresividad tienen una menor confianza con sus padres que aquellos/as que no muestran agresividad, con un tamaño medio del efecto. Los/as estudiantes que muestran mayores niveles de agresividad también muestran mayor alienación respecto a su padre que aquellos/as con niveles bajos de agresividad, con un tamaño alto del efecto (véase Tabla 3). No se encontraron diferencias en la agresividad en función de los niveles de comunicación que se presentan con el padre. 
Tabla 3

Puntuaciones en la Escala IPPA (padre) según agresividad.

\begin{tabular}{ccccc}
\hline & \multicolumn{2}{c}{ Confianza con padre } & \multicolumn{2}{c}{ Alienación padre } \\
\hline & Agresivos & No agresivos & Agresivos & No agresivos \\
\hline Media & 36.1 & 40.45 & 12.58 & 8.84 \\
DT & 8.1 & 4.74 & 4.6 & 3.07 \\
F & \multicolumn{2}{c}{$\mathrm{F}(68)=7.8$} & $\mathrm{~F}(68)=4.9$ \\
P & \multicolumn{2}{c}{$<5$} & $<.05$ \\
d de Cohen & & .66 & .96 \\
\hline
\end{tabular}

En relación a la madre, los/as estudiantes con mayores niveles de agresividad muestran mayor alienación respecto a su madre que los/as estudiantes con niveles bajos de agresividad, con un tamaño medio del efecto (véase Tabla 4). No se encontraron diferencias en la agresividad en función de los niveles de comunicación y confianza que se presentan con la madre.

Tabla 4

Puntuaciones en la Escala IPPA (madre) según agresividad.

\begin{tabular}{|c|c|c|}
\hline \multicolumn{3}{|c|}{ Alienación madre } \\
\hline & Agresivos & No agresivos \\
\hline Media & 12.2 & 9.27 \\
\hline DT & 4.01 & 4.24 \\
\hline $\mathrm{F}$ & \multicolumn{2}{|c|}{$F(110)=.124$} \\
\hline p & \multicolumn{2}{|c|}{$<.05$} \\
\hline d de Cohen & \multicolumn{2}{|c|}{.71} \\
\hline
\end{tabular}

Con respecto a la Valoración de la Gravedad de las Conductas de Agresión, los/as estudiantes que muestran un mayor nivel de agresión tienden a valorar las conductas agresivas como menos graves que aqueIlos/as que muestran menor nivel de agresión. Las diferencias de valoración son mayores en relación a conductas como dar collejas, gritar, tirar algún objeto de la clase a un/a compañero/a, etc. En aquellas conductas que pueden considerarse más graves, hay mayor grado de acuerdo respecto a su gravedad entre quienes presentan un nivel alto de agresividad y quienes muestran agresividad media y baja (véase Tabla 5). 
Tabla 5

Puntuaciones en Valoración de Gravedad en Conductas de Agresión

\begin{tabular}{|c|c|c|c|c|}
\hline \multicolumn{5}{|c|}{ Porcentaje de estudiantes } \\
\hline & CONDUCTAS & $\begin{array}{c}\text { Nada } \\
\text { o poco } \\
\text { grave }\end{array}$ & $\begin{array}{l}\text { Modera- } \\
\text { damente } \\
\text { grave }\end{array}$ & $\begin{array}{l}\text { Grave } \\
\text { o muy } \\
\text { grave }\end{array}$ \\
\hline \multirow{3}{*}{$\begin{array}{l}\text { Conductas } \\
\text { más graves }\end{array}$} & $\begin{array}{c}\text { Amenazarle para que haga cosas que } \\
\text { no quiere (dar dinero, etc.) }\end{array}$ & 2.8 & 3.3 & 93.5 \\
\hline & $\begin{array}{l}\text { Hacerle fotos o grabarle con el móvil } \\
\text { mientras se meten con él/ella }\end{array}$ & 7 & 0,9 & 92 \\
\hline & Romperle o robarle sus pertenencias & 2.8 & 7.9 & 89.3 \\
\hline \multirow{3}{*}{$\begin{array}{c}\text { Conductas } \\
\text { menos } \\
\text { graves }\end{array}$} & Tirarle algún objeto de la clase & 30.6 & 30.7 & 38.7 \\
\hline & Gritarle & 40.4 & 26.3 & 33.3 \\
\hline & Darle collejas & 48.2 & 25.2 & 26.7 \\
\hline
\end{tabular}

\section{Discusión y conclusiones}

Si bien el porcentaje de alumnos/as que se encuentra involucrado en conductas de agresión en el contexto escolar no es elevado, no deja de ser preocupante. Son numerosos los estudios que plantean el análisis de las causas de este fenómeno, unos destacan los factores de tipo personal y otros del contexto, ya sea escolar o familiar (Prodócimo, Cerezo y Arense, 2014). Continuar con esta línea de trabajo es necesario ya que puede ayudar en la creación de protocolos de prevención y actuación ante el acoso escolar.

Es de destacar que son los varones quienes se muestran más proclives a realizar comportamientos de acoso; lo cual confirma los resultados obtenidos en estudios previos (García-Moral, Gómez-Veiga, SánchezQueija, 2016; Giménez, Ballester, Dolores, Castro y Díaz, 2014; Matalinares, Arenas, Sotelo, Díaz, Dioses, Yaringaño, Muratta, Pareja y Tipacti, 2010; Postigo, González, Mateu, Ferrero y Martorell, 2009; Serrano e Iborra, 2005). Esta diferencia de género puede ser explicada por la influencia del estereotipo de masculinidad relacionado con el dominio y la fuerza (Díaz-Aguado, 2005; Santana, 2002, 2008a, 2008b). Las mujeres tienen mejores habilidades sociales y muestran mayores niveles de empatía y de preocupación por los/as otros/as; mientras que los varones manifiestan mayor desajuste social y mayor conducta antisocial (Giménez et al., 2014). En el acoso escolar, la violencia es horizontal -entre 
iguales-, pero en ella subyacen jerarquías sociales, en las que es determinante el sistema sexo/género y sus normas (Shute, Owens \& Slee, 2008).

El alumnado con padres de menor nivel educativo está más implicado en conductas agresivas. Resulta atrayente estudiar la vinculación de este resultado con la existente relación entre la incapacidad de los padres de regular emociones, y los problemas externalizantes asociados a la agresividad y el comportamiento social inapropiado de los/as hijos/ as (Eisenberg, Fabes, Guthrie \& Reiser, 2000).

Diversos estudios coindicen en que los/as estudiantes que han repetido algún curso en Educación Secundaria muestran una mayor frecuencia en la realización de conductas de acoso (Enríquez, 2015; Medel, 2017; Sánchez, 2009). El alumnado repetidor interpreta la situación de estar escolarizado en un grupo de edad inferior como una superioridad de edad y de fortaleza física (Cerezo, 2002). Además, tiende a presentar una baja integración social en el nuevo grupo de referencia, siendo más rechazado y aislado en el aula (Sánchez, 2009). Esto, según Melero (1993), puede ser debido a falta de motivación, dificultades de aprendizaje y/o una actitud negativa hacia la escuela.

La confianza en la relación con el padre tiene un papel importante para el alumnado agresor. Algunos estudios defienden que las relaciones con los padres son mejores predictoras de la agresividad de sus hijos/as que las relaciones con las madres (García-Moral et al., 2016). La calidad de la comunicación y la confianza que mantienen los estudiantes con sus madres no es determinante para el rol de agresión. La mayoría de los estudiantes tienen una buena comunicación con sus madres y confían en ellas, independientemente de su situación en las estructuras relacionales de las aulas. Sin embargo, algunos estudios han primado el análisis del papel del estilo educativo materno en el ajuste de los/as hijos/as, bajo la premisa de que la educación de los/as hijos/as recae en las madres (García-Moral et al., 2016).

El nivel de alienación se basa en los sentimientos de aislamiento que sienten los/as hijos/as respecto a sus progenitores. El nivel de alienación manifestado con padres y madres provoca mayores niveles de agresión, por lo que el mayor aislamiento social del alumnado se relaciona con una mayor vulnerabilidad al acoso escolar (Cava, Buelga, Musitu y Murgui, 2010). Esto nos acerca al conocimiento de los aspectos de la vida familiar que debemos trabajar con el alumnado con problemas de convivencia en la escuela. 
La familia constituye una estructura relacional cuya dinámica puede aumentar el comportamiento violento de los/as adolescentes, o disminuirlo (Prodócimo et al., 2014). Las relaciones seguras y confortables con los padres y las madres potencian en los/as hijos/as el aprendizaje de comportamientos positivos como la pro-socialidad o la empatía, e inhiben comportamientos sociales negativos como la agresividad (Farrant, Devine, Maybery \& Fletcher, 2012; Stanik, Riina \& McHale, 2013). La calidad de las relaciones familiares puede desembocar en una menor vulnerabilidad de los/as hijos/as, incrementar las competencias sociales, mejorar la resiliencia y reducir el acoso psicológico, la ansiedad y la depresión (Comisión de las Comunidades Europeas, 2005). Estos resultados nos permiten concluir que determinadas características socio-familiares actúan entre los/as jóvenes como factores de protección ante el acoso escolar. Entre ellas, se distinguen: la confianza que tienen los adolescentes con sus padres y el sentimiento de integración que proporcionan ambos progenitores, evitando la sensación de aislamiento de sus hijos/as.

Los estudiantes que realizan más comportamientos de acoso en la escuela valoran las conductas de agresión como menos graves que quienes muestran menores niveles de agresión. Esta diferencia es especialmente reveladora en las conductas menos peligrosas. Diversos estudios (Díaz-Aguado, 2005; Nolasco, 2012; Olweus, 1998; Serrano e Iborra, 2005) destacan la falta de empatía y autocrítica de los/as agresores/as ante las conductas de acoso. Los resultados encontrados invitan a plantear si la menor gravedad atribuida por los/as acosadores/ as a las conductas de agresión es debida a que: 1) tras la realización de conductas de acoso, tiene lugar una sucesión de distorsiones cognitivas, normalmente basadas en la culpabilización de la víctima, que "excusan, justifican o minimizan la gravedad del comportamiento" (Cepeda y Ruíz, 2016, p. 144); o a que 2) no considerar este tipo de comportamientos como especialmente graves es la causa de que los Ileven a cabo en el ámbito escolar.

En cuanto a las limitaciones del estudio, cabe destacar que se cuenta con un tamaño de la muestra limitado. Debido al interés de los resultados obtenidos, se considera conveniente replicar esta investigación con un número de participantes más amplio que permita establecer generalizaciones con mayor certeza. Es necesario examinar las diferencias que el alumnado presenta en los distintos factores de agresión según las variables socio-familiares y socio- afectivas, tanto desde una perspectiva 
cuantitativa, como desde un enfoque cualitativo a través de grupos de discusión.

Los resultados encontrados suponen un punto de partida, pero no podemos obviar los interrogantes que se abren para próximas investigaciones: a) ¿cuáles son las causas de que el acoso escolar esté presente en la mayoría de las escuelas?, b) ¿favorecen necesariamente los padres con bajo nivel educativo la aparición de conductas agresivas en sus hijos/as?, c) ¿por qué los/as repetidores/as presentan comportamientos de acoso escolar de manera más frecuente?, d) ¿por qué los padres tienen mayor influencia en las conductas de acoso escolar que las madres?, e) ¿la valoración de las conductas de agresión como menos graves por parte del alumnado agresor es una causa o consecuencia de esta mayor conflictividad? y, finalmente, f) ¿cómo podemos trabajar con las familias para disminuir la presencia de conflictos en la escuela? Es importante continuar con esta línea de investigación, ya que puede abrir camino en la eliminación o, al menos, en la reducción de las situaciones de acoso en los contextos escolares.

\section{Referencias}

Araujo, R., Gomes, T., Da Luz, L. y Medeiros, M. (2012). Reflexiones sobre la adolescencia y las funciones parentales en la realidad contemporánea. Aperturas psicoanalíticas, 41, 1-12.

Balan, R., Dobrean, A. \& Balazsi, R. (2018). Indirect effects of parental and peer attachment on bullying and victimization among adolescents: The role of negative automatic thoughts. Aggressive Behavior, 44(6), 561-570. doi:10.1002/ab.21775

Bowes, L., Arseneault, L., Maughan, B., Taylor, A., Caspi, A. \& Moffitt, T. E. (2009). School, neighborhood, and family factors are associated with children's bullying involvement: A nationally representative longitudinal study. Journal of the American Academy of Child \& Adolescent Psychiatry, 48(5), 545-553. doi:10.1097/CHI.0b013e31819cb017

Cantón, J. y Cortés, M. R. (2000) El apego del niño a sus cuidadores. Madrid: Alianza.

Castillo-Pulido, L. E. (2011). El acoso escolar. De las causas, origen y manifestaciones a la pregunta por el sentido que le otorgan los actores. Magis. Revista Internacional de Investigación en Educación, 4(8), 415-428.

Cava, M. J., Buelga, S., Musitu, G. y Murgui, S. (2010). Violencia escolar entre adolescentes y sus implicaciones en el ajuste psicosocial: un estudio longitudinal. Revista de Psicodidáctica, 15(1), 21-34.

Cepeda, Z. Y. y Ruiz, J. I. (2016). Distorsiones cognitivas: diferencias entre abusadores sexuales, delincuentes violentos y un grupo control. Revista Criminalidad, 58(2), 141 156. 
Acoso escolar y apego familiar: la perspectiva del alumnado agresor Iratxe Urresti-Padrón, Luis Feliciano-García y Lidia E. Santana-Vega

Cerezo, F. (2002). Conductas agresivas en la edad escolar. Aproximación teórica y metodología: propuestas de intervención. Madrid: Pirámide.

Comisión de las Comunidades Europeas (2005). Libro verde: mejorar la salud mental de la población. Hacia una estrategia de la unión europea en materia de salud mental. Bruselas: Comisión de las Comunidades Europeas. Recuperado de https://ec.europa. eu/health/ph_determinants/life_style/mental/green_paper/mental_gp_e s.pdf

Cooper, M. L., Shaver, P. R. \& Collins, N. L. (1998). Attachment styles, emotion regulation, and adjustment in adolescence. Journal of Personality and Social Psychology, 74(5), 1380-1397. doi:10.1037\%2F0022-3514.74.5.1380

De la Villa, M. y Ovejero, A. (2013). Percepción del clima social familiar y actitudes ante el acoso escolar en adolescentes. European Journal of Investigation in Health, Psychology and Education, 3(2), 149-160. doi:10.1989/ejihpe.v3i2.32

Demby, K. P., Riggs, S. A. \& Kaminski, P. L. (2017). Attachment and Family Processes in Children's Psychological Adjustment in Middle Childhood. Family Process, 56(1), 234-249. doi:10.1111/famp.12145

Díaz-Aguado, M. J. (2005). La violencia entre iguales en la adolescencia y su prevención desde la escuela. Psicothema, 17(4), 549-558.

Eisenberg, N., Fabes, R. A., Guthrie, I. K. \& Reiser, M. (2000). Dispositional emotionality and regulation: Their role in predicting quality of social functioning. Journal of Personality and Social Psychology, 78(1), 136-157. doi:10.1037//0022-3514.78.1.136

Enríquez, D. (2015). Análisis de la evolución del acoso escolar: factores, rol e incidencia de las redes sociales y contexto familiar en IES de Málaga (Tesis doctoral, Universidad de Málaga).

Espelage, D. L. \& Swearer, S. M. (2009). Contributions of three social theories to understanding bullying perpetration and victimization among school-aged youth. En M. Harris (Ed.), Bullying, rejection \& peer victimization. A Social Cognitive Neuroscience perspective (pp. 151-170). New York: Springer.

Espelage, D. L., Bosworth, K. \& Simon, T. R. (2000). Examining the social context of buIlying behaviors in early adolescence. Journal of Counseling and Development, 78, 326-333. doi:10.1002/j.1556-6676.2000.tb01914.x

Falla, D. y Ortega-Ruiz, R. (2019). Los Escolares Diagnosticados con Trastorno del Espectro Autista y Víctimas de Acoso Escolar: una Revisión Sistemática. Psicología Educativa, 25(2), 77-90. doi:10.5093/psed2019a6

Farrant, B. M., Devine, T. A. J., Maybery, M. T. \& Fletcher, J. (2012). Empathy, perspective taking and prosocial behaviour: the important of parenting practices. Infant and Child Development, 21(2), 175-188. doi:10.1002/icd.740

Fernández, O., Goñi, E., Camino, I. y Ramos, E. (2019). Apoyo social percibido e implicación escolar del alumnado de educación secundaria. Revista Española de Pedagogía, 77(272), 123-141. doi:10.22550/REP77-1-2019-06

García-Moral, A. T., Gómez-Veiga, I. y Sánchez-Queija, I. (2016). Efecto diferencial del estilo educativo paterno y materno en la agresividad durante la adolescencia. Behavioral Psychology/Psicología Conductual, 24(3), 497-511.

Georgiou, S. N. \& Fanti, K. A. (2010). A transactional model of bullying and victimization. Social Psychology of Education, 13(3), 295-311. doi:10.1007\%2Fs11218-010-9116-0 
Giménez, C., Ballester, R., Dolores, M., Castro, J. y Díaz, I. (2014). Roles de género y agresividad en la adolescencia. International Journal of Developmental and Educational Psychology, 2(1), 373-382. doi:10.17060/ijodaep.2014.n1.v2.452

Gómez-Ortiz, O., Del Rey, R., Casas, J. A. y Ortega-Ruiz, R. (2014). Parenting styles and bullying involvement / Estilos parentales e implicación en bullying. Cultura y Educación, 26(1), 132-158. doi:10.1080/11356405.2014.908665

Gómez-Ortiz, O., Del Rey, R., Romera, E. M. y Ortega-Ruiz, R. (2015). Los estilos educativos paternos y maternos en la adolescencia y su relación con la resiliencia, el apego y la implicación en acoso escolar. Anales de Psicología, 31(3), 979-989. doi:10.6018/ analesps.31.3.180791

González-Cabrera, J., Tourón, J., Machimbarrena, J. M., León-Mejía, A. y Gutiérrez-Ortega, M. (2019). Estudio exploratorio sobre acoso escolar en alumnado con altas capacidades: prevalencia y afectación psicológica. Revista de Educación, 386, 187- 214. doi:10.4438/1988-592X-RE-2019-386-432

Hong, J. S., Kim, D. H. \& Piquero, A. R. (2017). Assessing the links between punitive parenting, peer deviance, social isolation and bullying perpetration and victimization in South Korean adolescents. Child Abuse \& Neglect, 73, 63-70. doi:10.1016/j. chiabu.2017.09.017

Low, S. \& Espelage, D. L. (2013). Differentiating cyberbullying perpetration from other forms of peer aggression: Commonalities across race, individual, and family predictors. Psychology of Violence, 3(1), 39-52. doi:10.1037/a0030308

Magaz, A. M., Chorot, P., Sandin, B., Santed, M. A. y Valiente, R. M. (2011). Estilo de apego y acoso entre iguales (bullying) en adolescentes. Revista de Psicopatología y Psicología Clínica, 16(3), 207-221. doi:10.5944/rppc.vol.16.num.3.2011.10362

Mann, M. J., Kristjansson, A. L., Sigfusdottir, I. D. \& Smith, M. L. (2015). The Role of Community, Family, Peer, and School Factors in Group Bullying: Implications for School-Based Intervention. Journal of School Health, 85(7), 477-486. doi:10.1111/ josh. 12270

Matalinares, M., Arenas, C., Sotelo, L., Díaz, G., Dioses, A., Yaringaño, J., Muratta, R., Pareja, C. y Tipacti, R. (2010). Clima familiar y agresividad en estudiantes de secundaria de lima metropolitana. Revista IIPSI, 13(1), 109-128. doi:10.15381/rinvp. v13i1.3740

Medel, M. (2017). Inteligencia emocional y acoso escolar en ESO (Tesis doctoral, Universidad de Huelva).

Melero (1993). Conflictividad y violencia en los centros escolares. Madrid: Siglo XXI. Ministerio de Educación, Cultura y Deporte (2019). Acoso escolar, datos, cifras y estadísticas.

España: Epdata. Recuperado de https://www.epdata.es/datos/acoso-escolar-datos- cifrasestadisticas/257/espana/106

Murphy, T. P., Laible, D. \& Augustine, M. (2017). The Influences of Parent and Peer Attachment on Bullying. Journal of Child and Family Studies, 26(5), 1388-1397. doi:10.1007/s10826-017-0663-2

Nolasco, A. (2012). La empatía y su relación con el acoso escolar. Revista de Estudios y Experiencias en Educación, 11(22), 35-54. 
Acoso escolar y apego familiar: la perspectiva del alumnado agresor Iratxe Urresti-Padrón, Luis Feliciano-García y Lidia E. Santana-Vega

Nuñez, M. C., Herrero, S. y Aires, M. M. (2006). Diez referencias destacadas sobre el acoso escolar. Anuario de Psicología Clínica y de la Salud, 2, 35-50.

Olweus, D. (1997). Bully/victim problems in school: Facts and intervention. European Journal of Psychology of Education, 12(495), 495-510. doi:10.1007/bf03172807

Olweus, D. (1998). Conductas de acoso y amenaza entre escolares. Madrid: Ediciones Morata, S.L.

Oñate, A. y Piñuel, I. (2007). Acoso y Violencia Escolar en España: Informe Cisneros X. Madrid: Instituto de Innovación Educativa y Desarrollo Directivo.

Pellegrini, A. D. \& Long, J. D (2002). A longitudinal study of bullying, dominance, and victimization during the transition from primary school through secondary school. British Journal of Developmental Psychology, 20(2), 259-280. doi:10.1348/026151002166442

Postigo, S., González, R., Mateu, C., Ferrero, J. y Martorell, C. (2009). Diferencias conductuales según género en convivencia escolar. Psicothema, 21(3), 453-458.

Prodócimo, E., Cerezo, F. y Arense, J. J. (2014). Acoso escolar: variables sociofamiliares como factores de riesgo o de protección. Behavioral Psychology/Psicología Conductual, 22(2), 343-357.

Pulido, L. y Tarancón, P. (2018). Opiniones y experiencias respecto al bullying: estudio cualitativo en un Instituto de Educación Secundaria de la ciudad de Albacete. ENSAYOS, Revista de la Facultad de Educación de Albacete, 33(2), 29-45.

Rodrigo, M. J., Máiquez, M. L., Batista-Foguet, J. M., García, M., Rodríguez, G., Martín, U. C. y Martínez, A. (2006). Estilos de vida en la adolescencia y su relación con los contextos de desarrollo. Cultura y Educación, 18(3-4), 381-395. doi: $10.1174 / 113564006779173064$

Sánchez, C. (2009). Nivel de implicación en bullying entre escolares de educación primaria. Relación con el estatus sociométrico y la percepción del clima social, familiar y escolar (Tesis doctoral, Universidad de Murcia).

Santana, L. E. (2002). Mujeres, igualdad de oportunidades y transición sociolaboral. Revista de Educación, 327, 169-188.

Santana, L. E. (2008a). Educar en la etapa de secundaria. En L. E. Santana Vega (Coord.). Educar en secundaria. Retos de la tutoría (pp. 25-58). Madrid: Wolters Kluwer.

Santana, L. E. (2008b). Las dos mitades de la humanidad: la igualdad efectiva de mujeres y hombres. En C. Jiménez y G. Serrano (Coords.). Educación y género, el conocimiento invisible (pp. 73-97). Valencia: Tirant Lo Blanch.

Santana, L. E. (2013). Orientación Profesional. Madrid: Síntesis.

Santana, L. E., Feliciano, L. y Jiménez, A. B. (2012). Toma de decisiones y género en Bachillerato. Revista de Educación, 359, 357-387. doi:10.4438/1988-592X-RE-2011359-098

Serrano, Á. e Iborra, I. (2005). Informe Violencia entre compañeros en la escuela. Valencia: Centro Reina Sofía para el Estudio de la Violencia.

Shetgiri, R., Lin, H., Avila, R. M. \& Flores, G. (2012). Parental characteristics associated with bullying perpetration in US children aged 10 to 17 years. American Journal of Public Health, 102(12), 2280-2286. doi:10.2105/AJPH.2012.300725

Shute, R., Owens, L. \& Slee, P. (2008). Everyday victimization of adolescent girls by boys: 
Acoso escolar y apego familiar: la perspectiva del alumnado agresor

Iratxe Urresti-Padrón, Luis Feliciano-García y Lidia E. Santana-Vega

Sexual harassment, bullying or aggression? Sex Roles, 58(7-8), 477-489. doi:10.1007/ s11199-007-9363-5

Stanik, C. E., Riina, E. M. \& McHale, S. M. (2013). Parent-adolescent relationship qualities and adolescent adjustment in two-parent African American families. Family Relations, 62(4), 597-608. doi:10.1111/fare.12020

Ulloa, L. (2015). Preconcepciones de maltrato escolar y su relación con autoestima, autoeficacia y apoyo social en escolares agresores y víctimas de ambos sexos, de edades entre 12 y 19 años de colegios subvencionados de la ciudad de viña del mar-chile (Tesis doctoral, Universidad de Málaga).

Valdés, Á., Martínez, E. y Torres, G. (2012). Diferencias en la situación socioeconómica, clima y ajuste familiar de estudiantes con reportes de bullying y sin ellos. Psicología desde el Caribe, 29(3), 616-631.

You, S., Lee, J., Lee. Y. \& Kim, A. Y. (2015). Bullying among korean adolescents: the role of empathy and attachment. Psychology in the Schools, 52(6), 594-606. doi:10.1002/ pits. 21842 
